\title{
Inhibition kinetics and molecular simulation of $p$-substituted cinnamic acid derivatives on tyrosinase
}

Yi Cui ${ }^{1}$, Yong-Hua $\mathrm{Hu}^{1}$, Feng $\mathrm{Yu}^{1}$, Jing Zheng ${ }^{1}$, Lin-Shan Chen ${ }^{1}$, Qing-Xi Chen ${ }^{1,2}$,

Qin Wang ${ }^{1} *$

${ }^{1}$ Key Laboratory of the Ministry of Education for Coastal and Wetland Ecosystems,

Xiamen University, School of Life Sciences, Xiamen 361005, China,

${ }^{2}$ Key Laboratory for Chemical Biology of Fujian Province, Xiamen University,

Xiamen 361005, China

* Authors for corresponding. Tel/fax: +86 592 2184648. E-mail: qwang@xmu.edu.cn_ 


\section{ABSTRACT}

This study was to investigate the inhibition effects of para-substituted cinnamic acid derivatives (4-chlorocinnamic acid, 4-ethoxycinnamic acid and 4-nitrocinnamic acid) on tyrosinase catalyzing the substrates, with the purpose of elucidating the inhibition mechanism of the tested derivatives on tyrosinase by the UV-Vis spectrum, fluorescence spectroscopy, copper interacting and molecular docking, respectively. The native-PAGE results showed that 4-chlorocinnamic acid (4-CCA), 4-ethoxycinnamic acid (4-ECA) and 4-nitrocinnamic acid (4-NCA) had inhibitory effects on tyrosinase. Spectrophotometric analysis used to determine the inhibition capabilities of these compounds on tyrosinase catalyzing L-tyrosine (L-Tyr) and L-3,4-Dihydroxyphenylalanine (L-DOPA) as well. The $I C_{50}$ values and inhibition constants were further determined. Moreover, quenching mechanisms of tested compounds to tyrosinase belonged to static type and a red shift on fluorescence emission peak occurred when 4-NCA added. Copper interacting and molecular docking demonstrated that 4-CCA could not bind directly to the copper, but it could interact with residues in the active center of tyrosinase. Meanwhile, 4-ECA and 4-NCA could chelate a copper ion of tyrosinase. Anti-tyrosinase activities of para-substituted cinnamic acid derivatives would lay scientific foundation for their utilization in designing of novel tyrosinase inhibitors.

Keywords: derivatives of cinnamic acid; fluorescence spectra; molecular simulation 


\section{Introduction}

Tyrosinase (EC 1.14.18.1) is a multifunctional copper-containing enzyme that is important for melanin biosynthesis in plants and animals [1]. It catalyzes the hydroxylation of tyrosine (monophenol) to L-DOPA (o-diphenol), and the oxidation of L-DOPA to its corresponding derivative (o-quinone) [2], which is further converted into melanin. Since tyrosinase plays an important role in biosynthesizing melanin, the enzyme is usually associated with pigmentation. Therefore, tyrosinase inhibitors have potential applications in medicine, cosmetics to prevent hyperpigmentation by inhibiting enzymatic oxidation [3]. Structurally, the binuclear active site of tyrosinase was composed of two copper ions, coordinated each by three highly conserved histidines [4]. These copper ions were directly involved in the process of enzymatic oxidation [3].

Large numbers of tyrosinase inhibitors have been reported and most of them consist of a phenol structure or of metal chelating agents [5-9]. Our front-stage work had found that cinnamic acid was an effective tyrosinase inhibitor with $I C_{50}=2.10 \mathrm{mM}$ [10]. Cinnamic acid is one of the major components of Cinnamomum cassia BLUME, and is a wildly distributed phenylpropanoid component naturally occurring in plants [11]. This compound is reported to have the depigmenting ability and effects on the expression of melanin biosynthesis-related enzymes [12]. In the search for tyrosinase inhibitors, cinnamic acid is a substrate analogue, and owing to this chemical aspect cinnamic acid derivatives received much attention. As reported by Okombi et al., 4-hydroxycinnamic acid derivatives were able to inhibit melanin synthesis through inhibition of tyrosinase [13]. Lee et al. found that 3,4-dihydroxycinnamic acid and 4-hydroxy-3-methoxycinnamic acid exhibited potent antityrosinase activity, and methoxy substitutions, such as 2-methoxycinnamic acid, 3-methoxycinnamic acid, 
and 4-methoxycinnamic acid could enhance inhibition of tyrosinase activity [14].

In our previous study, we found that 4-hydroxycinnamic acid and 4-methoxycinnamic acid strongly inhibited the diphenolase activity of mushroom tyrosinase with $I C_{50}$ of 0.50 and $0.42 \mathrm{mM}$, respectively, whose inhibitory effects were much better than cinnamic acid [10]. In this work, we chose three para-substituted cinnamic acid derivatives (4-CCA, 4-ECA and 4-NCA) to investigate the inhibitory kinetics and molecular simulation on mushroom tyrosinase. The knowledge offered in this manuscript should help to provide leads to the ultimate goal of developing new tyrosinase inhibitors of adequate efficacy for the prevention of browning.

\section{Materials and methods}

\subsection{Materials}

4-CCA, 4-ECA and 4-NCA were purchased from Aladdin Reagent (Shanghai, China). Mushroom tyrosinase (EC 1.14.18.1) with activity of 6680U/mg, L-DOPA and L-Tyr were the products of Sigma-Aldrich (St. Louis, MO). Coomassie brilliant blue was bought at Nanjing Jiancheng Bioengineering Institute (Nanjing, Jiangsu, China). Other reagents were obtained from Sinopharm (Shanghai, China) with standard analytical purity. The water used was redistilled and ion-free.

\subsection{Enzyme activity assay}

The monophenolase and diphenolase activity of tyrosinase were measured by following the absorbance at $475 \mathrm{~nm}\left(\varepsilon=3700 \mathrm{M}^{-1} \mathrm{~cm}^{-1}\right)$ with the oxidation of L-Tyr and L-DOPA, respectively, using a Beckman DU800 spectrophotometer [15]. $3 \mathrm{~mL}$ system contained $1 \mathrm{mM}$ L-Tyr or $0.5 \mathrm{mM}$ L-DOPA and $50 \mu \mathrm{L}$ enzyme in $50 \mathrm{mM}$ $\mathrm{NaH}_{2} \mathrm{PO}_{4}-\mathrm{Na}_{2} \mathrm{HPO}_{4}$ buffer, $\mathrm{pH}$ 6.8. The inhibitory type and constants were obtained by the previously reported methods [16]. 


\subsection{Protein electrophoresis}

Native-PAGE was used to identify the interaction between tyrosinase and inhibitor [17]. The final tyrosinase activity was $1000 \mathrm{U} / \mathrm{mL}$ in sample. After electrophoresis, the gels were cut into a piece each lane and soaked in inhibitors with different concentrations for $30 \mathrm{~min}$, and then were incubated in $0.5 \mathrm{mM} \mathrm{L}-\mathrm{DOPA}$ at $37^{\circ} \mathrm{C}$ for 5 min for vital staining. Except vital staining, the other process was conducted in $4{ }^{\circ} \mathrm{C}$. Bands were quantified using the ImageJ 1.48 software after scanning of the pages.

\subsection{Scanning study}

The assay was operated according to the method described by Jiménez-Atiénzar et al. with slight modifications [18]. $1 \mathrm{mM}$ L-Tyr or $0.5 \mathrm{mM}$ L-DOPA was added to $3 \mathrm{~mL}$ reaction media, which contained $0.1 \mathrm{~mL}$ inhibitor in $50 \mathrm{mM} \mathrm{Na} 2 \mathrm{HPO}_{4}-\mathrm{NaH}_{2} \mathrm{PO}_{4}$ buffer (pH 6.8). The final concentration of tyrosinase was $16.67 \mu \mathrm{g} / \mathrm{mL}$. Before and after inhibitor was added, the time-dependent UV-visible spectra of the solution were monitored at wavelengths from 240 to $700 \mathrm{~nm}$. All samples were recorded at room temperature using a Beckman UV-800 spectrophotometer.

\subsection{Fluorescence spectra quenching}

Fluorescence spectra were performed with reference to Kim et al [19], and the experiment was recorded at $25^{\circ} \mathrm{C}$ on a Cary Eclipse fluorescence spectrophotometer with an excitation wavelength $\left(\lambda_{\mathrm{ex}}\right)$ of $280 \mathrm{~nm}$. Fluorescence emission spectra were recorded at wavelengths of 300-420 nm [20-22]. In this assay, $10 \mu \mathrm{L}$ inhibitors with different concentrations were added to $2 \mathrm{~mL}$ tyrosinase solution, and the final concentration of enzyme was $0.2 \mathrm{mg} / \mathrm{mL}$. Fluorescence quenching was described by the Stern-Volmer equation [23, 24]:

$$
\mathrm{F}_{0} / \mathrm{F}=1+K_{\mathrm{q}} \tau_{0}[\mathrm{I}]=1+K_{\mathrm{SV}}[\mathrm{I}]
$$


Where $F_{0}$ and $F$ are the fluorescence intensities without and with the quencher, respectively; $K_{\mathrm{q}}$ is the bimolecular quenching constant; $\tau_{0}\left(10^{-8} \mathrm{~s}\right)$ is the lifetime of the fluorophore when the quencher exist [25]; [I] is the quencher concentration and $K_{\mathrm{SV}}$ is the Stern-Volmer quenching constant.

Static quenching procedure can be described by Scatchard equation to estimate the apparent binding constant $\left(K_{\mathrm{A}}\right)$ and the binding affinity $(n)$ as follows [26]:

$$
\lg \left[\left(\mathrm{F}_{0}-\mathrm{F}\right) / \mathrm{F}\right]=\lg K_{\mathrm{A}}+n \lg [\mathrm{I}]
$$

\subsection{Copper Interaction}

To investigate the interaction between the copper ions and three compounds, copper sulphate $\left(\mathrm{CuSO}_{4}\right)$ was added to the assay medium. The concentrations of three inhibitors were $0.25 \mathrm{mM}$. The absorbances at $475 \mathrm{~nm}$ were taken after the addition of different concentration of $\mathrm{CuSO}_{4}$ to the samples using a DU800 spectrophotometer [27].

\subsection{Molecular docking simulation in silico}

The molecular operation environment 2010 software (MOE) was used to simulate docking of protein and ligand with the method described by Chen [28]. The following steps were taken for docking and simulation: (1) minimizing energy of the 3D structures of inhibitors and tyrosinase, (2) identifying the active site of tyrosinase, (3) docking tyrosinase and inhibitor, (4) simulating molecular dynamics. For the in silico protein-ligand docking, the parameters were set as follows: the refinement was set to force field; the retain of both the first and second scoring were set to 20; the rescoring were set to London $\mathrm{dG}$; the MM/GBVI binding free energy scoring was used to rank the docking poses. A more negative value indicates a stronger interaction. Other parameters used were the default settings of the software. The docked conformation, which had the highest score, was selected to analyze the mode of binding. 


\section{Results}

3.1. Inhibitory effects of derivatives of cinnamic acid on the monophenolase activity of mushroom tyrosinase

The inhibitory effects of 4-CCA, 4-ECA and 4-NCA with different concentrations on the oxidation of L-Tyr by mushroom tyrosinase were studied. From the progress curves of the oxidation of L-Tyr, the lag times and monophenolase activities were observed. After the lag time, the system reached the steady-state [29]. The kinetic courses were shown in Fig. 1A-a, Fig. 1B-a and Fig. 1C-a. With the increasing concentrations of three compounds, the lag times became longer (Fig. 1A-b, Fig. 1B-b and Fig. 1C-b) and the steady-state rates decreased distinctly and dose-dependently (Fig. 1A-c, Fig. 1B-c and Fig. 1C-c). The concentration leading to 50\% loss of enzyme activity $\left(I C_{50}\right)$ for 4-CCA, 4-ECA and 4-NCA was measured to be 0.477 , 0.980 and $0.521 \mathrm{mM}$, respectively. The values were shown in Table 1 as well. When the concentration of inhibitor reached $0.6 \mathrm{mM}$, the lag time could be delayed by 4-CCA, 4-ECA and 4-NCA up to $130.2 \%, 116.2 \%$ and $42.5 \%$, respectively. The results indicated that all the inhibitor in test can affect both lag time and steady-state rate of tyrosinase, and 4-CCA had a best inhibitory effect among them.

\subsection{Inhibitory effects of derivatives of cinnamic acid on the diphenolase activity.}

Intuitively, 4-CCA, 4-ECA and 4-NCA can inhibit tyrosinase activity, similar to the reports for a series of derivatives of cinnamic acid and the supposition was tested based on the previously established method [10]. Three derivatives significantly inhibited the diphenolase activity in a dose-dependent manner (Fig. 2A-a, Fig. 2B-a and Fig. 2C-a). The $I C_{50}$ values of 4-CCA, 4-ECA and 4-NCA were determined to be $0.229,0.252$ and $0.318 \mathrm{mM}$, respectively. 
The inhibition mechanisms of these three derivatives were investigated. Fig. 2A-b showed that the plots of the residual enzyme activity versus the enzyme concentrations at different concentrations of 4-CCA gave a series of straight lines, which all passed through the origin. With the increase of the inhibitor concentration, the slope of the line descended indicating that the inhibitory mechanism by 4-CCA on diphenolase was reversible. The inhibition of 4-ECA and 4-NCA on tyrosinase followed the same mechanism (Fig. 2B-b and Fig. 2C-b).

A kinetic study was conducted to explore the inhibition type of 4-CCA by Lineweaver-Burk plot analysis as shown in Fig. 2A-c-I indicating that 4-CCA was a noncompetitive inhibitor. Fig. 2B-c-I and Fig. 2C-c-I suggested that both 4-ECA and 4-NCA were mixed type inhibitors of tyrosinase.

The inhibition constant $\left(K_{\mathrm{I}}\right)$ of 4-CCA calculated to be $0.227 \mathrm{mM}$ from Fig. 2A-c-II, and the value of enzyme-substrate complex $\left(K_{\mathrm{IS}}\right)$ equaled to $K_{\mathrm{I}}$. For 4 -ECA and 4-NCA, the values of $K_{\mathrm{I}}$ were $0.216 \mathrm{mM}$ and $0.211 \mathrm{mM}$, which were acquired from the plots of the slopes versus inhibitor concentrations (Fig. 2B-c-II and Fig. 2C-c-II), and the values of $K_{\text {IS }}$ obtained from the vertical intercepts versus inhibitor concentrations were determined to be $0.451 \mathrm{mM}$ and $1.192 \mathrm{mM}$ (Fig. 2B-c-III and Fig. 2C-c-III). The related values were listed in Table 1 for comparison.

\subsection{Native-PAGE electrophoresis analysis}

Tyrosinase contained at least two parts with different molecular weights, which were usually $42-46 \mathrm{kDa}[30,31]$ and $64-68 \mathrm{kDa}[31]$. As Fig. 3A shown, tyrosinase stained with Coomassie brilliant blue (lane 1) and L-DOPA (lane 2) showed both two molecular weight ranges existed in the sample and were active. 
Native-PAGE electrophoresis is another method to study the inhibition effect of inhibitors on tyrosinase [17]. Fig. 3B and Fig. 3C showed that the tyrosinase activity staining bands were clearly reduced when the concentration of compounds increased, especially for the ranges of 42-46 kDa. When the concentration of 4-CCA, 4-ECA and 4-NCA reached $20 \mathrm{mM}$, the band of 42-46 kDa were almost disappeared. Further, the bands of 42-46 kDa were disappeared when inhibitors' concentrations reached $40 \mathrm{mM}$. The results showed that the para-substituted cinnamic acid derivatives in test could inhibit the activity of tyrosinase.

\subsection{Effects on oxidation of L-Tyr/L-DOPA by the derivatives of cinnamic acid}

UV-visible spectra of enzymatically oxidized L-Tyr and L-DOPA were exhibited in Fig. 4. The peaks at 303 and $475 \mathrm{~nm}$ suggested the formation of dopachrome $\left(\lambda_{\max }\right.$ $=302,473 \mathrm{~nm}$ ) [32]. When L-Tyr was used as a substrate, the product spectra with or without inhibitors showed an intensive absorbance at $475 \mathrm{~nm}$, but the intensities of the $A_{475 \mathrm{~nm}}$ were different (Fig. 4A). After 10 minutes, the presence of 4-CCA, 4-ECA and 4-NCA reduced the peak intensity by $47.83 \%, 30.37 \%$ and $22.97 \%$, respectively. Similarly, as shown in Fig. 4B, the oxidation of L-DOPA inhibited by 4-CCA, 4-ECA and $4-\mathrm{NCA}$ were $46.95 \%, 34.99 \%$ and $32.84 \%$, respectively, at $5 \mathrm{~min}$ after the addition of the enzyme.

\subsection{Analysis of fluorescence quenching}

When 4-CCA or 4-ECA was mixed with tyrosinase, the fluorescence of mixture was depicted in Fig. 5A-a and Fig. 5B-a. With the concentration increasing, both 4-CCA and 4-ECA had a quenching effect on the intrinsic fluorescence without significant $\lambda_{\mathrm{em}}$ shift (Fig. 5A-b and Fig. 5B-b), indicating that 4-CCA or 4-ECA bound to tyrosinase 
depends on the concentration only. Moreover, Fig. 5C-a showed the fluorescence intensity of tyrosinase decreased with increasing 4-NCA concentration, and the maximum emission wavelength was changed from 337 to $356 \mathrm{~nm}$ (Fig. 5C-b). The red shift signified that tyrosinase conformation was changed.

The plots of $\mathrm{F}_{0} / \mathrm{F}$ against the concentrations of quenchers were showed in Fig. 5A-d, Fig. 5B-d and Fig. 5C-d. $K_{\mathrm{SV}}$ could be obtained from the slope of regression curves, and $K_{\mathrm{q}}$ were calculated based on lifetime of the fluorophore $\left(\tau_{0}\right)$. All the values were shown in Table 2.

The maximum diffusion collision quenching rate constant of various quenchers with the biopolymer is $K_{\mathrm{q}}{ }^{\prime}=2.0 \times 10^{10} \mathrm{M}^{-1} \mathrm{~s}^{-1}$ [22]. In Table 2 , the values of $K_{\mathrm{q}}$ of all derivatives to tyrosinase were far larger than the $K_{\mathrm{q}}{ }^{\prime}$. Therefore, the quenching should follow a static quenching process. For static quenching, Scatchard equation was used to study the relationship of the fluorescence intensity and the concentrations of quenchers. As shown in Table 2, binding constants decreased in the order: 4-ECA > 4-CCA > 4-NCA.

\subsection{The chelating copper ions ability}

Many tyrosinase inhibitors that chelate the copper ions in the active site of the enzyme showed good inhibition effects; examples included tropolone [33] and kojic acid [34]. To investigate whether the tested compounds exerted its inhibitory effect by chelating the copper ions at the active site of tyrosinase, copper sulphate $\left(\mathrm{CuSO}_{4}\right)$ was added to the assay medium. As shown in Fig. 6A-a, tyrosinase activity was decreased with the increasing of $\mathrm{CuSO}_{4}$, indicating that 4-CCA had no ability to chelate the copper. However, when $\mathrm{CuSO}_{4}$ was added to the assay medium which contained 4-ECA or 4-NCA, an optimum $\mathrm{CuSO}_{4}$ concentration of $30 \mu \mathrm{M}$ was able to partially prevent the 
inhibition of tyrosinase by $0.25 \mathrm{mM}$ inhibitor (about 50\% of total activity) (Fig. 6A-B and Fig. 6A-c). The results may due to the formation of copper-4-ECA and copper-4-NCA complex did not affect tyrosinase activity. Lower $\mathrm{CuSO}_{4}$ concentrations were less effective to prevent the inhibition and an excess of copper could inhibit tyrosinase activity as well [35].

3.7. Molecular modeling for the binding of the derivatives of cinnamic acid with tyrosinase

The diagrammatic sketch depicting the interaction of ligand with the protein was obtained by using MOE software. As shown in Fig. 6B-a, 4-CCA docked onto the active site of tyrosinase. The interactions between 4-CCA with the active site residues were observed but without interaction with copper ions. The docked 4-CCA surrounded by eleven residues, namely, His61, His85, His259, Asn260, His263, Phe264, Met 280, Ser 282, Val 283, Phe292 and His296. It can be seen clearly in Fig. 6B-b that 4-ECA docked inside the binding region of tyrosinase. The oxygen atom of oxethyl formed metal contact with one copper ion and interact with the residue His296. Moreover, one interaction of 4-ECA with the residue Asn260 was found with the hydroxyl group of carboxyl. The same analysis was carried out in Fig. 6B-c, which indicated that one copper ion formed two metal contacts with 4-NCA and one with the residue His61. Two residues (His259 and His296) could directly act on the oxygen atom of carboxyl. Other residues that found in the vicinity of 4-NCA were His85, His94, Asn260, His263, Phe264, Val283 and Phe292. The above results were consistent with the results of the chelating copper ions ability assay.

\section{Discussion}

The anti-tyrosinase activity assays revealed that three derivatives of cinnamic acid had 
inhibitory effects on monophenolase activity for both lag time and steady-state rate. This mode of inhibition was also reported by $\mathrm{Hu}$ et al. in their investigation of the inhibition kinetics of 2-chlorcinnamic acid and 2,4-dichlorocinnamic acid on the enzyme[36]. The effects of three compounds on the diphenolase activities of mushroom tyrosinase were studied by two methods. The results of enzyme assay indicated that the $I C_{50}$ values of them were similar (Table 1), which was consistent with native-PAGE electrophoresis. However, the inhibition mechanisms of them were different, which may be caused by the structural difference of three compounds on substituent at the para-position. As for 4-CCA, it showed a non-competitive inhibition pattern, which indicated that 4-CCA had no opportunity to inhibit tyrosinase directly. Perhaps because chloridion could not interdict the oxy-state for generating a lower affinity to copper ions than oxygen. The similar results have been reported by Park et al. as well [37]. Moreover, the inhibition mechanisms of 4-ECA and 4-NCA were mixed type. It was suggested that they could form a tight complex with tyrosinase and it substrate. The tight complex could make the free oxygen molecule unable to take part in the hydroxylation with monophenols and the oxidation with $o$-diphenols. Therefore tyrosinase would lose its catalyzing ability.

The results of fluorescence spectroscopy showed that all tested compounds could decrease tyrosinase fluorescence intensity. However, no apparent $\lambda_{\mathrm{em}}$ shift in the fluorescence emission spectra with the addition of 4-CCA and 4-ECA. The results indicated that the interaction between 4-CCA or 4-ECA and tyrosinase just affected the microenvironment in close proximity to the fluorophore of tyrosinase without changes in the immediate surroundings of the quenched residues. The same phenomenon also be found by $\mathrm{Mu}$ et al. in the study of tyrosinase and tricin [38]. Nevertheless, 4-NCA quenched fluorescence with an obvious red shift in peak 
wavelength from 337 to $356 \mathrm{~nm}$. The shift was attributed to an increased polarity (or a decreased hydrophobicity) of the region surrounding the active site of tyrosinase. The nitro group of 4-NCA could make the red shifts of fluorescence spectra as it had a strong electron-donating ability and increased polarity of the region surrounding the active site of tyrosinase. $\mathrm{Xu}$ et al. also found that the electron-donating ability of the two $\mathrm{N}$ atoms on 1,8-naphthalimide could make red shifts in both absorption and fluorescence spectra [39].

Computational docking was used to predict the reactions between ligands and tyrosinase. The results showed that 4-CCA could interact with residues near the active site without binding directly to the copper ions in the active center of tyrosinase. The similar examples of such inhibitors was $\alpha$-bromocinnamaldehyde [16], which could occupy the position of $\mathrm{CuA}$ to suppress the monophenolase activity. Moreover, 4-ECA and 4-NCA could not only interact with residues near the active site but also form metal interactions with a copper ion of the enzyme. As shown in result, the group of 4-NCA combined with copper ion was carboxyl, which was different from 4-ECA. It was speculated that the strong electron-withdrawing nitro group of 4-NCA could cause steric or electrostatic effect which may impact the geometry of tyrosinase. In conclusion, as an anti-tyrosinase agent, the presence of the substituent at the para-position were approximately ten times more effective than cinnamic acid with an $I C_{50}$ of $2.10 \mathrm{mM}$ [10]. The above findings demonstrated that substituent group at para-position may enhance the inhibition effect. Walker et al. suggested that there were two distinct sites on the enzyme: one side for the binding of the substrate and another, adjacent site for binding to the inhibitor [40]. We hypothesize that these three derivatives of cinnamic acid were attached to a site different from the active site, and they hindered the binding of substrate to the enzyme through steric hindrance or by 
changing the protein conformation.

\title{
Acknowledgements
}

The present investigation was supported by the Natural Science Foundation of China (Grant No.31271952 and 31570785), the Fundamental Research Funds for the Central Universities (Grant No. 20720140541) and by the National Science Foundation for Fostering Talents in Basic Research of the National Natural Science Foundation of China (Grant No. J1310027).

\author{
Abbreviations \\ 4-CCA, 4-chlorocinnamic acid; 4-ECA, 4-ethoxycinnamic acid; 4-NCA, \\ 4-nitrocinnamic acid; DMSO, dimethyl-sulfoxide; L-Tyr, L-tyrosine ; L-DOPA, L-3, \\ 4-Dihydroxyphenylalanine; $K_{\mathrm{m}}$, Michaelis-Menten constant; $K_{\mathrm{I}}$, equilibrium constant \\ of the inhibitor combining with the free enzyme; $I C_{50}$, inhibitor concentration leading \\ to a $50 \%$ loss of activity
}




\section{References}

1. Y. J. Zhu, K. K. Song, Z. C. Li, Z. Z. Pan, Y. J. Guo, J. J. Zhou, Q. Wang, B. Liu, Q. X. Chen, J Agr Food Chem, 57 (2009) 5518-5523.

2. C. J. Cooksey, P. J. Garratt, E. J. Land, S. Pavel, C. A. Ramsden, P. A. Riley, N. P. M. Smit, J Biol Chem, 272 (1997) 26226-26235.

3. Y. X. Si, Z. J. Wang, D. Park, H. Y. Chung, S. F. Wang, L. Yan, J. M. Yang, G. Y. Qian, S. J. Yin, Y. D. Park, Int J Biol Macromol, 50 (2012) 257-262.

4. C. Gasparetti, E. Nordlund, J. Janis, J. Buchert, K. Kruus, Bba-Proteins Proteom, 1824 (2012) 598-607.

5. Y. Marrero-Ponce, G. M. Casanola-Martin, M. T. H. Khan, F. Torrens, A. Rescigno, C. Abad, Curr Pharm Design, 16 (2010) 2601-2624.

6. S. Parvez, M. Kang, H. S. Chung, H. Bae, Phytother Res, 21 (2007) 805-816.

7. H. Ando, H. Kondoh, M. Ichihashi, V. J. Hearing, J Invest Dermatol, 127 (2007) $751-761$.

8. Y. J. Kim, H. Uyama, Cell Mol Life Sci, 62 (2005) 1707-1723.

9. A. Karioti, A. Protopappa, N. Megoulas, H. Skaltsa, Bioorgan Med Chem, 15 (2007) $2708-2714$

10. Y. Shi, Q. X. Chen, Q. Wang, K. K. Song, L. Qiu, Food Chem, 92 (2005) 707-712.

11. Z. D. He, C. F. Qiao, Q. B. Han, C. L. Cheng, H. X. Xu, R. W. Jiang, P. P. H. But, P. C. Shaw, J Agr Food Chem, 53 (2005) 2424-2428.

12. Y. H. Kong, Y. O. Jo, C. W. Cho, D. Son, S. Park, J. Rho, S. Y. Choi, Biol Pharm Bull, 31 (2008) 946-954. 
13. S. Okombi, D. Rival, S. Bonnet, A. M. Mariotte, E. Perrier, A. Boumendjel, Bioorg Med Chem Lett, 16 (2006) 2252-2255.

14. H. S. Lee, J Agr Food Chem, 50 (2002) 1400-1403.

15. K. K. Song, Q. X. Chen, Q. Wang, L. Qiu, H. Huang, J Enzym Inhib Med Ch, 20 (2005) 239-243.

16. Y. Cui, G. Liang, Y. H. Hu, Y. Shi, Y. X. Cai, H. J. Gao, Q. X. Chen, Q. Wang, J Agr Food Chem, 63 (2015) 716-722.

[17] R. Xing, A.P. Zheng, F. Wang, L. Wang, Y.P. Yu, A.H. Jiang, Food Chem, 175 (2015) 292-299.

18. M. Jimenez-Atienzar, J. Cabanes, F. Gandia-Herrero, F. Garcia-Carmona, Biochem Bioph Res Co, 319 (2004) 902-910.

19. D. Kim, J. Park, J. Kim, C. Han, J. Yoon, N. Kim, J. Seo, C. Lee, J Agr Food Chem, 54 (2006) 935-941.

20. T. H. Zhu, S. W. Cao, Y. Y. Yu, Int J Biol Macromol, 62 (2013) 589-595.

21. J. N. Tian, J. Q. Liu, J. P. Xie, X. J. Yao, Z. D. Hu, X. G. Chen, J Photoch Photobio B, 74 (2004) 39-45.

22. H. R. Wang, W. J. Zhu, X. Y. Wang, Int J Biol Macromol, 49 (2011) 985-991.

23. H. N. Tian, J. Q. Liu, J. Y. Zhang, Z. D. Hu, X. G. Chen, Chem Pharm Bull, 51 (2003) 579-582.

24. Y. J. Hu, Y. Liu, T. Q. Sun, A. M. Bai, H. Q. Lu, Z. B. Pi, Int J Biol Macromol, 39 (2006) 280-285.

25. J. R. Lakowicz, G. Weber, Biochemistry-Us, 12 (1973) 4161-4170. 
26. Y. Zhao, Y. Cao, F. M. Han, Y. Chen, Spectrosc Spect Anal, 28 (2008) 904-907.

27. J. C. Espin, H. J. Wichers, Bba-Protein Struct M, 1544 (2001) 289-300.

28 X. X. Chen, J. Zhang, W. M. Chai, H. L. Feng, Z. H. Xiang, D. Y. Shen, Q. X. Chen, Int J Biol Macromol, 62 (2013) 726-733.

29. Z. C. Li, L. H. Chen, X. J. Yu, Y. H. Hu, K. K. Song, X. W. Zhou, Q. X. Chen, J Agr Food Chem, 58 (2010) 12537-12540.

30. C. Gerdemann, C. Eicken, B. Krebs, Accounts Chem Res, 35 (2002) 183-191.

31. H. J. Wichers, K. Recourt, M. Hendriks, C. E. M. Ebbelaar, G. Biancone, F. A. Hoeberichts, H. Mooibroek, C. Soler-Rivas, Appl Microbiol Biot, 61 (2003) $336-341$

32. B. P. Lee, J. L. Dalsin, P. B. Messersmith, Biomacromolecules, 3 (2002) 1038-1047.

33. S. M. Son, K. D. Moon, C. Y. Lee, J Agr Food Chem, 48 (2000) 2071-2074.

34. G. Battaini, E. Monzani, L. Casella, L. Santagostini, R. Pagliarin, J Biol Inorg Chem, 5 (2000) 262-268.

35. V. Kahn, A. Andrawis, Phytochemistry, 24 (1985) 905-908.

36. Y. H. Hu, X. Liu, Y. L. Jia, Y. J. Guo, Q. Wang, Q.X. Chen, J Biosci Bioeng, 117 (2014) 142-146.

37. Y. D. Park, S. Y. Kim, Y. J. Lyou, J. Y. Lee, J. M. Yang, Biochimie, 87 (2005) 931-937.

38. Y. Mu, L. Li, S. Q. Hu, Spectrochim Acta A, 107 (2013) 235-240.

39. Z. C. Xu, X. H. Qian, J. N. Cui, Org Lett, 7 (2005) 3029-3032. 
40. J. R. Walker, E. L. Wilson, J Sci Food Agr, 26 (1975) 1825-1831. 
Table 1. Inhibition constants of 4-CCA, 4-ECA and 4-NCA

\begin{tabular}{|c|c|c|c|c|c|c|c|}
\hline \multirow[t]{2}{*}{ Compd } & \multicolumn{2}{|c|}{$\begin{array}{c}\text { Monophenolase } \\
\text { activity }\end{array}$} & \multicolumn{3}{|c|}{ Diphenolase activity } & \multicolumn{2}{|c|}{$\begin{array}{l}\text { Inhibition } \\
\text { constants } \\
(\mathrm{mM})\end{array}$} \\
\hline & $\begin{array}{l}I C_{50} \\
(\mathrm{mM})\end{array}$ & $\begin{array}{c}\text { Lag } \\
\text { effect }\end{array}$ & $\begin{array}{l}I C_{50} \\
(\mathrm{mM})\end{array}$ & $\begin{array}{l}\text { Inhibitory } \\
\text { mechanism }\end{array}$ & Inhibitory type & $K_{\mathrm{I}}$ & $K_{\mathrm{IS}}$ \\
\hline 4-CCA & 0.477 & $130.2 \%$ & 0.229 & Reversible & Non-competitive & 0.227 & 0.227 \\
\hline 4-ECA & 0.980 & $116.2 \%$ & 0.252 & Reversible & mixed & 0.216 & 0.451 \\
\hline 4-NCA & 0.521 & $42.5 \%$ & 0.318 & Reversible & mixed & 0.211 & 1.192 \\
\hline
\end{tabular}


Table 2. Stern-Volmer Equation for the Interaction between quenchers and tyrosinase

\begin{tabular}{cllll}
\hline Compound & $K_{\mathrm{SV}}\left(\mathrm{M}^{-1}\right)$ & $K_{\mathrm{q}}\left(\mathrm{M}^{-1} \mathrm{~s}^{-1}\right)$ & $K_{\mathrm{A}}\left(\mathrm{M}^{-1}\right)$ & $n$ \\
\hline 4-CCA & $4.54 \times 10^{4}$ & $4.54 \times 10^{12}$ & $4.37 \times 10^{5}$ & 1.13 \\
4-ECA & $5.58 \times 10^{4}$ & $5.58 \times 10^{12}$ & $3.31 \times 10^{5}$ & 1.08 \\
$4-\mathrm{NCA}$ & $3.89 \times 10^{4}$ & $3.89 \times 10^{12}$ & $1.66 \times 10^{5}$ & 1.06 \\
\hline
\end{tabular}




\section{Figure caption}

Fig. 1. The inhibition of 4-CCA (A), 4-ECA (B) and 4-NCA (C) on monophenolase activities of mushroom tyrosinase. (a) Progress curves of the oxidation of L-Tyr; (b) Effects on the lag time; (c) Effects on the stable activity. The concentrations of 4-CCA for curves $1-6$ were $0,0.1,0.2,0.3,0.5$, and $0.7 \mathrm{mM}$. The concentrations of 4 -ECA and 4-NCA for curves $1-6$ were $0,0.2,0.4,0.6,0.8$, and $1.0 \mathrm{mM}$.

Fig. 2. The inhibition of 4-CCA (A), 4-ECA (B) and 4-NCA (C) on diphenolase activities of mushroom tyrosinase. (a) Inhibitory activities of the oxidation of L-DOPA; (b) Effects of tyrosinase concentrations on its diphenolase activities at different concentrations of inhibitors. The concentrations of 4-CCA, 4-ECA and 4-NCA for curves $1-5$ were $0,0.125,0.25,0.375$, and $0.5 \mathrm{mM}$. (c) The inhibition types and constants. The concentrations of 4-CCA for curves $1-5$ were $0,0.125,0.25$, 0.375, and $0.5 \mathrm{mM}$. The concentrations of 4-ECA for curves $1-5$ were $0,0.15,0.3$, 0.45 , and $0.6 \mathrm{mM}$. The concentrations of 4-NCA for curves $1-5$ were $0,0.2,0.4,0.6$, and $0.8 \mathrm{mM}$.

Fig. 3. Native-PAGE analysis of the inhibitory effects of 4-CCA, 4-ECA and 4-NCA on the diphenolase activities of mushroom tyrosinase. (A) Determination of the protein molecular weight and species of mushroom tyrosinase. M: protein molecular weight markers; 1: tyrosinase stained with Coomassie blue; 2: tyrosinase stained with L-DOPA. (B), (C) Inhibitory effects of 4-CCA (a), 4-ECA (b) and 4-NCA (c) on mushroom tyrosinase. The concentrations of inhibitors for lanes 1-5 were $0,5,10,20$ and $40 \mathrm{mM}$, respectively. 
Fig. 4. UV-Visible spectra for the oxidation of L-Tyr (A) and L-DOPA (B). (a) $1 \mathrm{mg} / \mathrm{mL}$ L-DOPA oxidated by tyrosinase; (b), (c) and (d) represent oxidation of L-DOPA at the presence of 4-CCA, 4-ECA and 4-NCA, respectively. The final concentration of inhibitor was $0.25 \mathrm{mM}$. Insets represent the subtraction of respective inhibitor spectrum. Lines 1-11 represent $0-10 \mathrm{~min}$ after the addition of the enzyme; Lines 1-5 represent 0-4 min after the addition of the enzyme.

Fig. 5. Changes in intrinsic tyrosinase fluorescence at different concentrations of 4-CCA (A), 4-ECA (B) and 4-NCA (C). (a) Intrinsic fluorescence changes, the concentrations of quenchers for lines 1-6 were $0,0.01,0.02,0.03,0.04$ and $0.05 \mathrm{mM}$, respectively. (b) Maximum peak wavelength changes; (c) Relative fluorescence intensity changes; (d) Stern-Volmer plot describing the tyrosinase quenching caused by association with quenchers; (e) Scatchard equation estimating the apparent binding constant and the binding affinity. F0 and F are the fluorescence intensities before and after the addition of quenchers.

Fig. 6. (A) Dependence of $\mathrm{CuSO} 4$ concentration versus tyrosinase activity inhibited by 4-CCA (a), 4-ECA (b) and 4-NCA (c). (B) Binding modes of 4-CCA (a), 4-ECA (b) and 4-NCA (c) with tyrosinase active sites. The molecular of inhibitors were shown as green, while the copper ions were yellow. 
Fig. 1
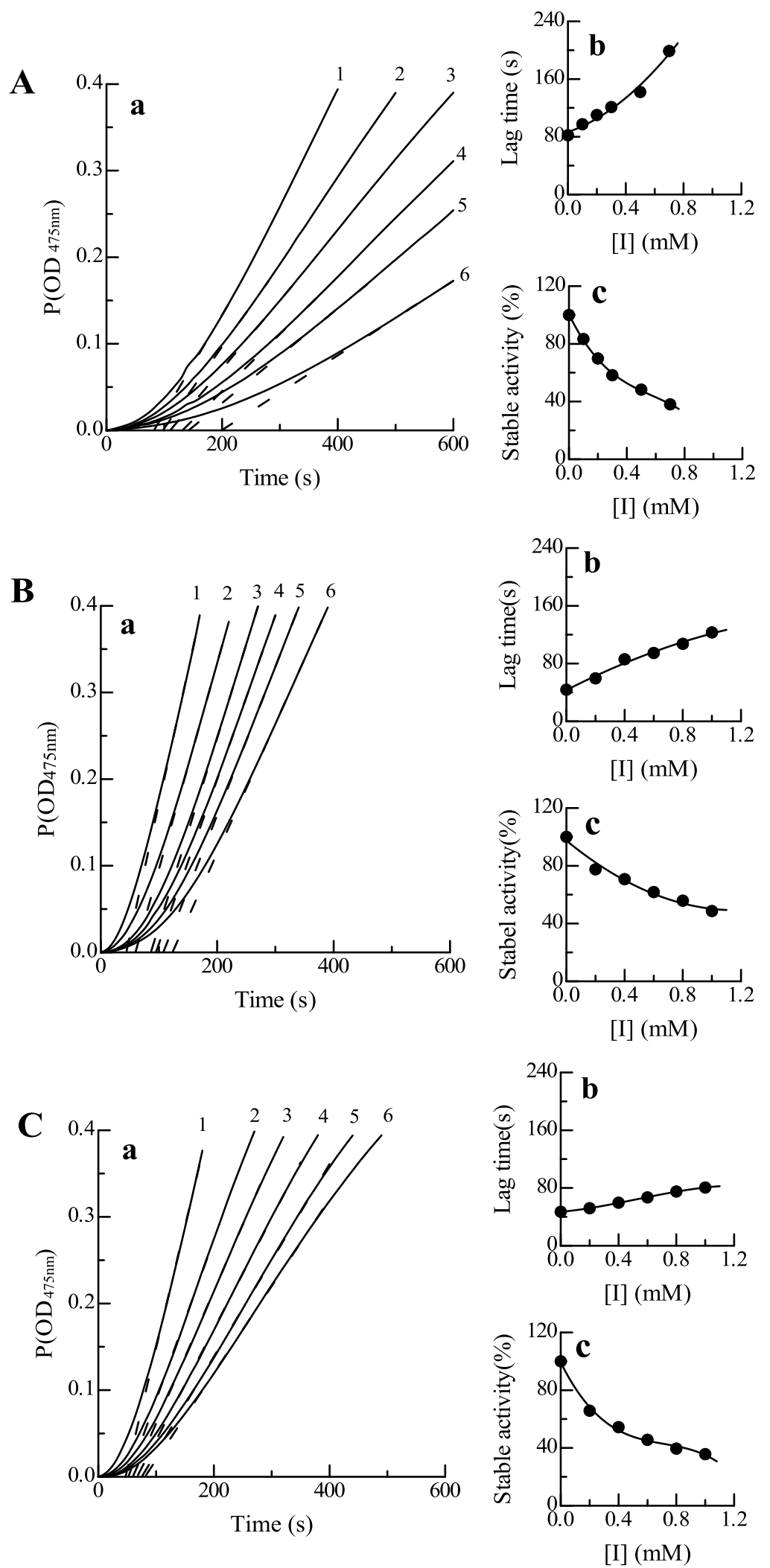
Fig. 2
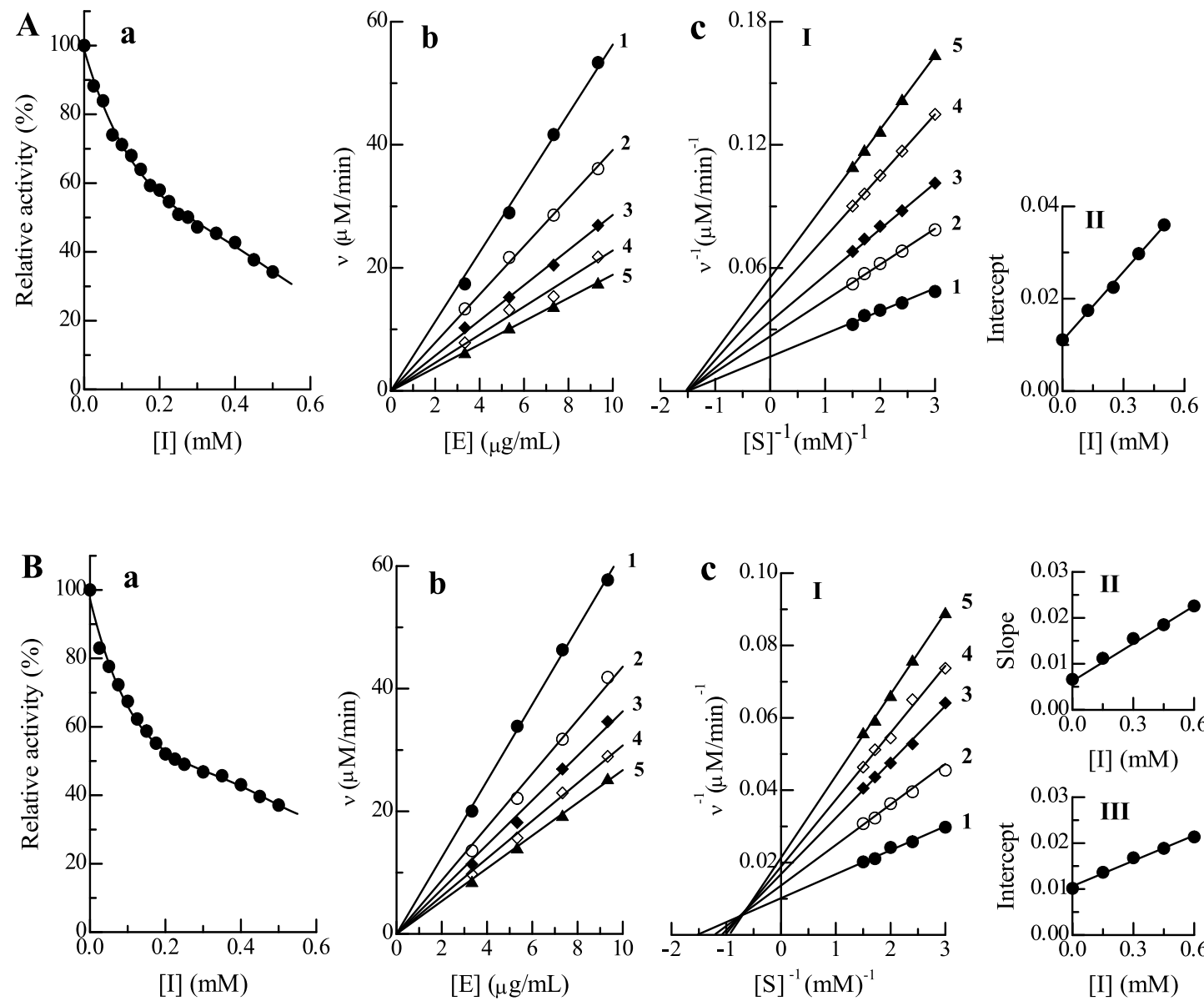

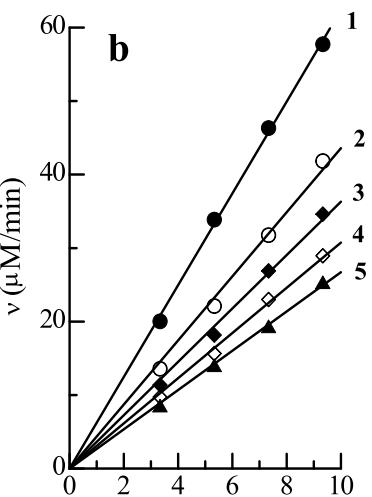

[E] $(\mu \mathrm{g} / \mathrm{mL})$

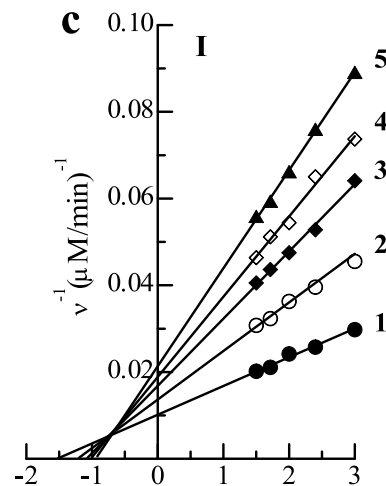

$[\mathrm{S}]^{-1}(\mathrm{mM})^{-1}$
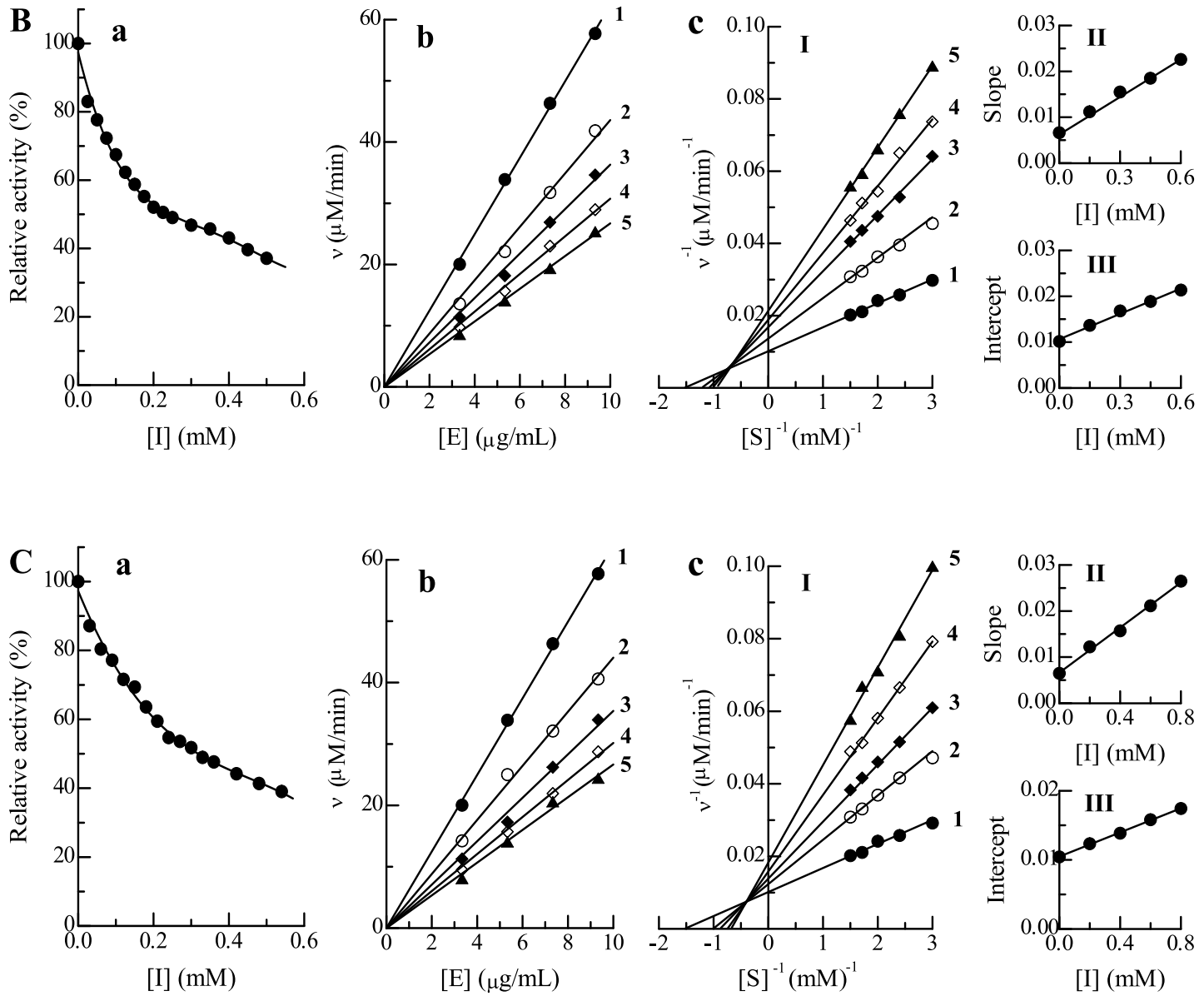
Fig. 3

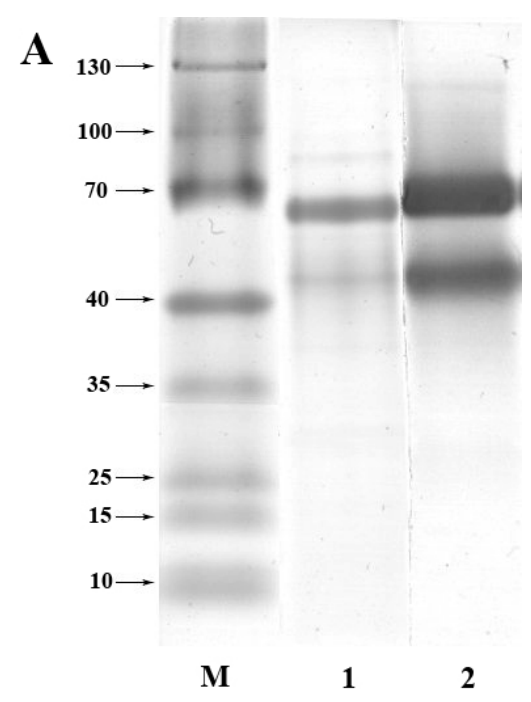

B a

b

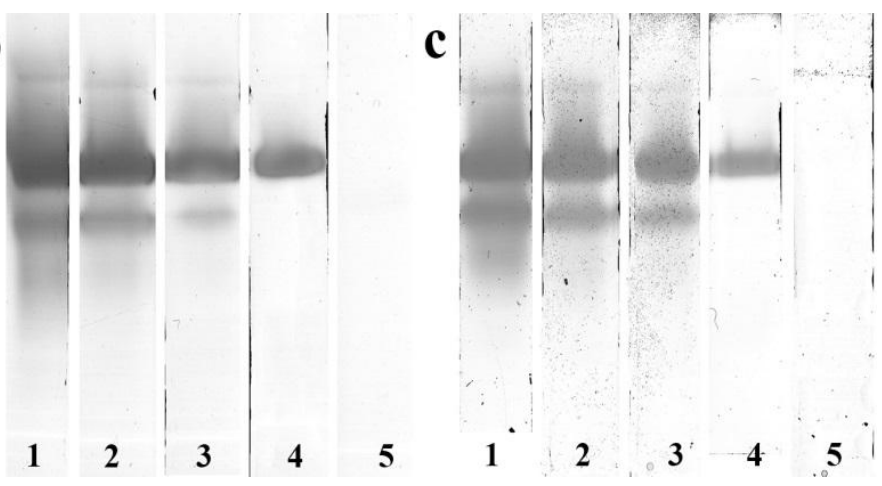

C
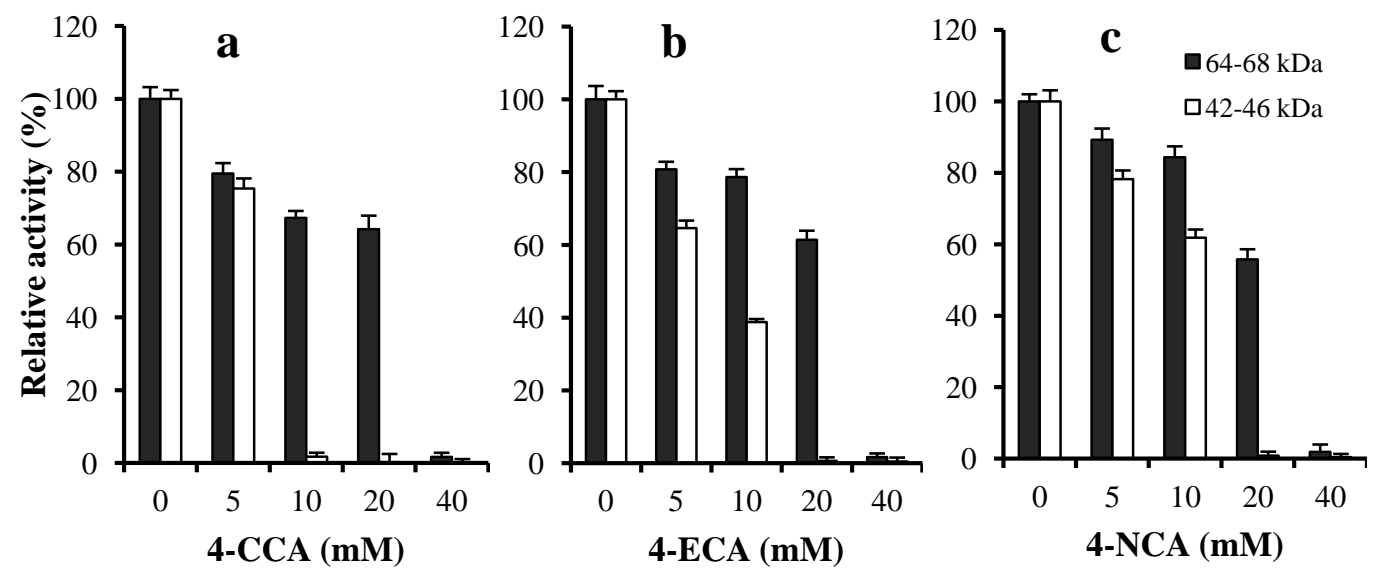
Fig. 4
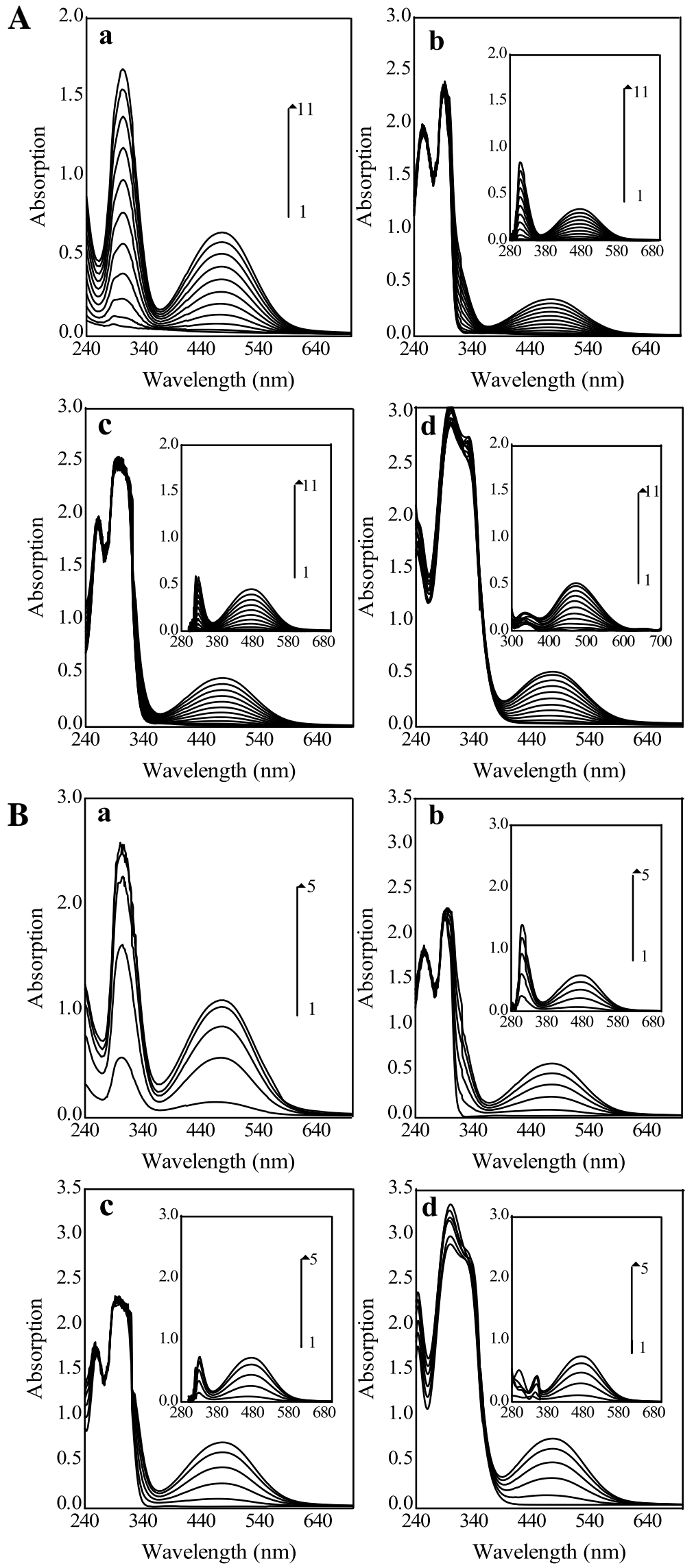
Fig. 5
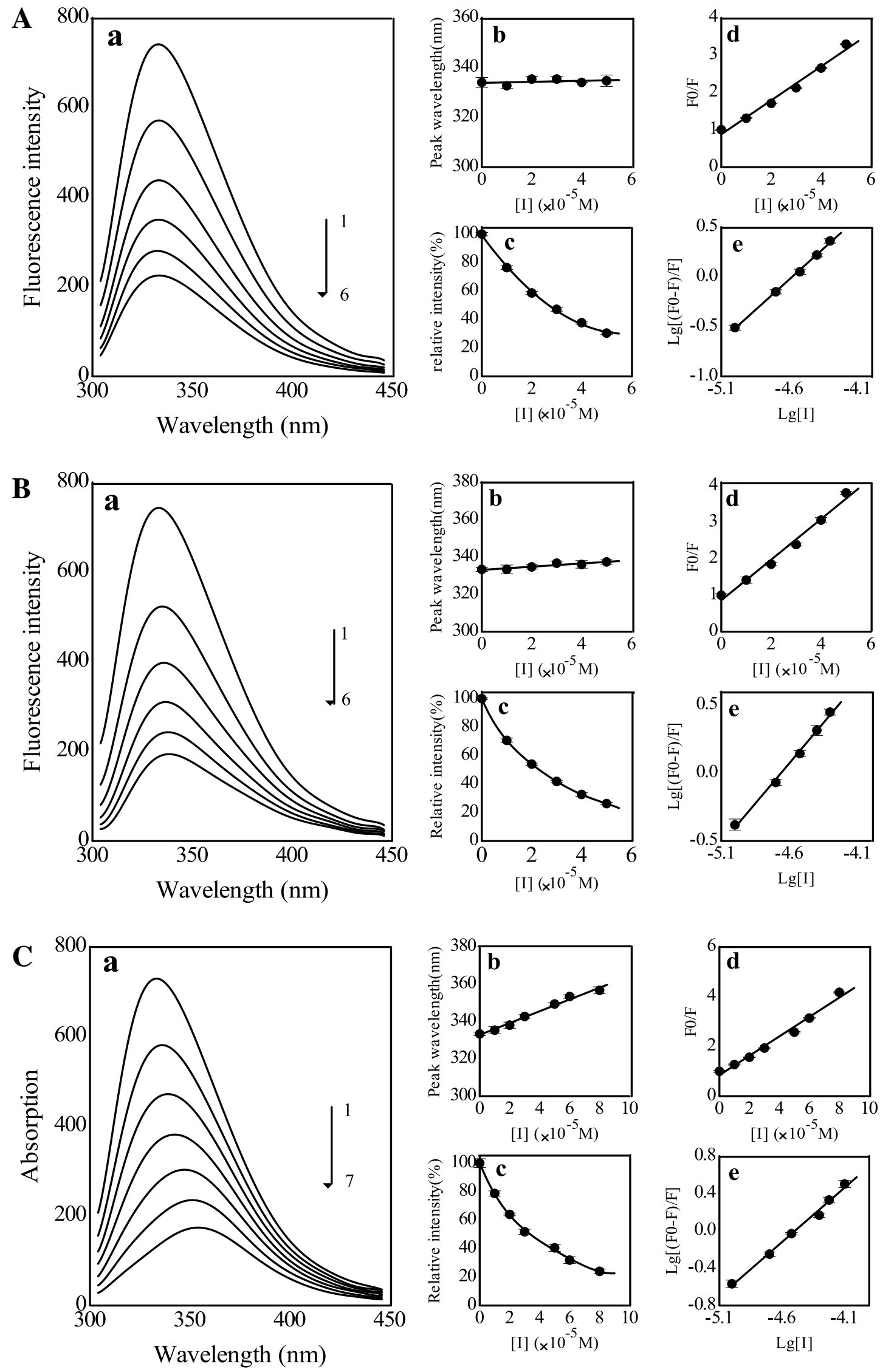
Fig. 6
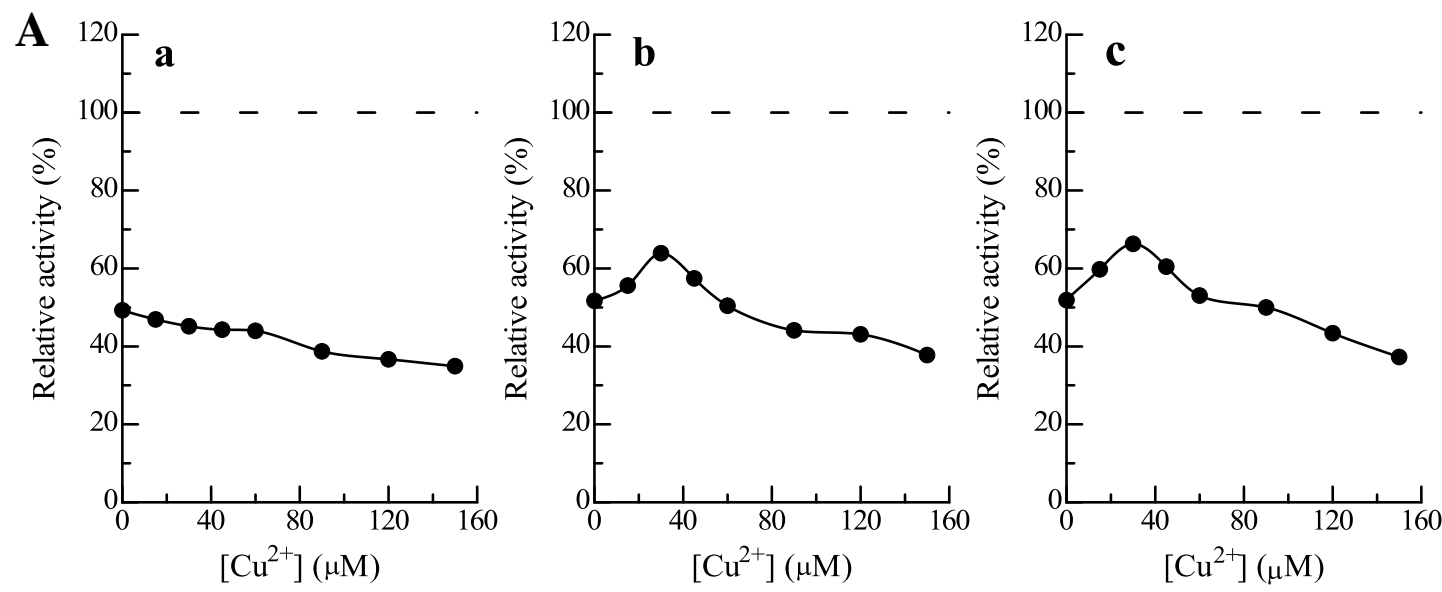

\section{B}
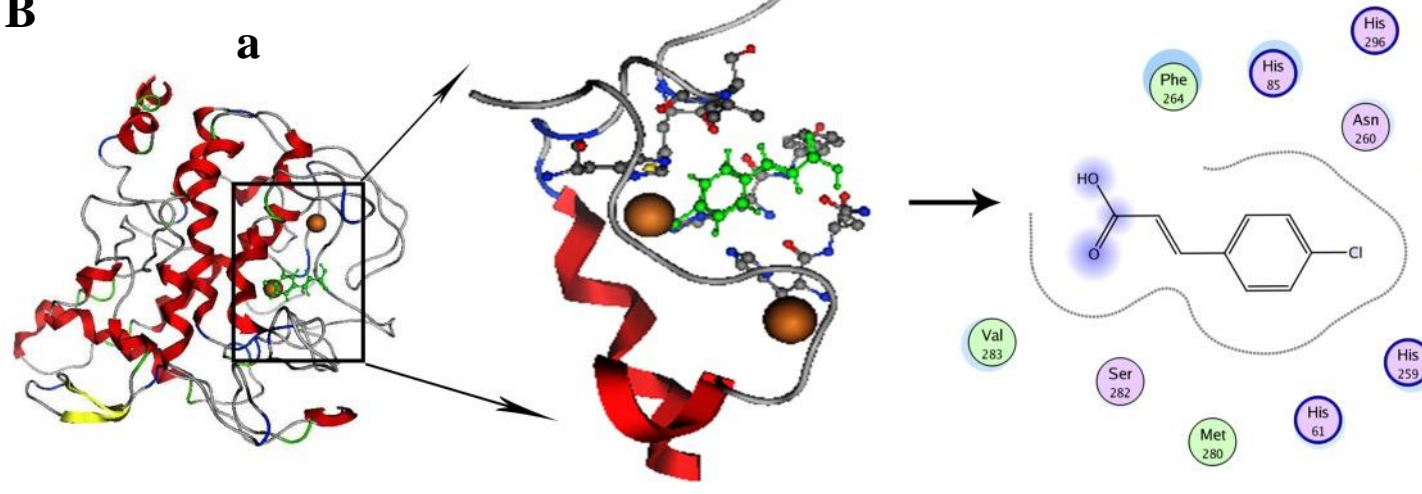

b

$\left(\begin{array}{c}\text { His } \\ 85\end{array}\right) \quad \begin{gathered}\text { Cys } \\ 83\end{gathered}$

c

$\left(\begin{array}{c}\mathrm{His} \\ 85\end{array}\right.$

Phe

(116) (116)

(His

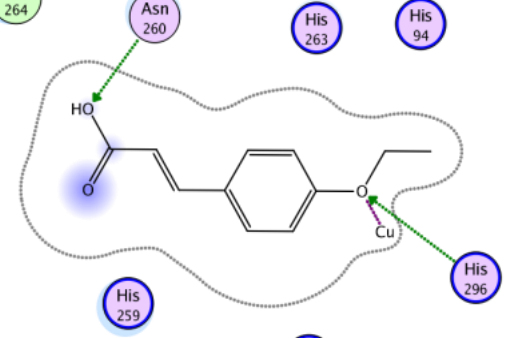

(is

Phe
292

Phe
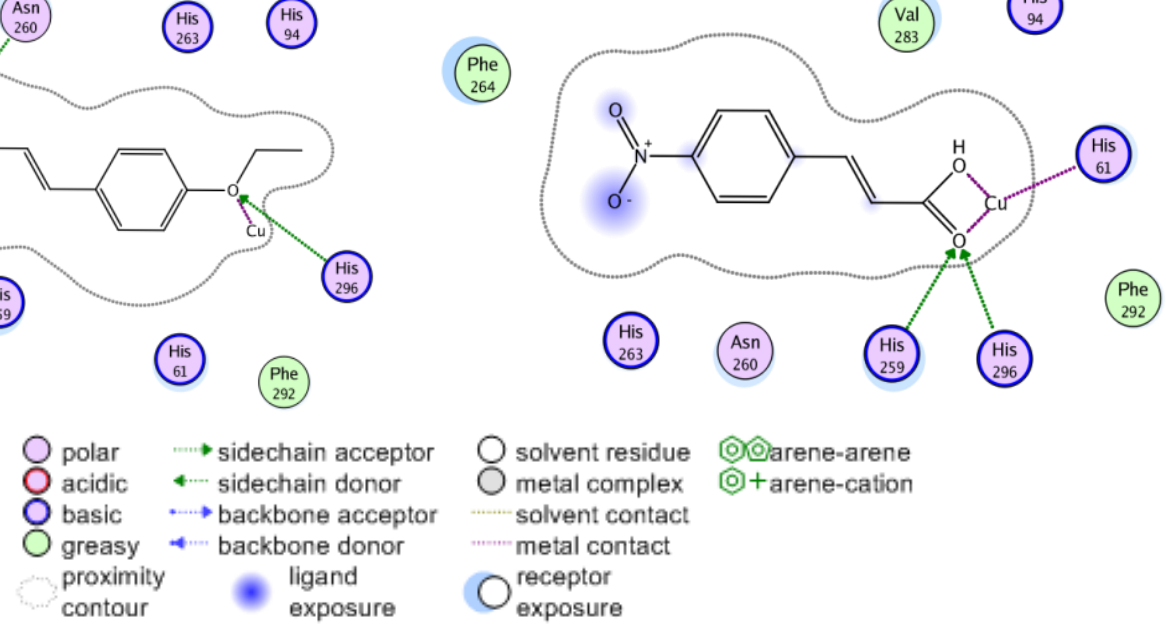

solvent residue

(O)

metal complex

O) +arene-cation

metal contact

$O_{\text {exposure }}^{\text {receptor }}$ 